\title{
Research on Japanese-original Anime Words based on Chinese Network Catchword Sphere
}

\author{
Jin Chang Cai \\ Teacher's College, Beihua University, Jilin, China
}

\begin{abstract}
In this paper, the phenomenon of Japanese-original anime language's spreading in Chinese network language was discussed, and some theory of lexicology, semantics, grammar, and cultural linguistics was proposed. The characters of its different types and its influence on Chinese and the reason why the anime language can be so popular in the factors of psychologies, social also was analyzed. According to these studies, some suggestions were put forward that how to standardize the network language and how to raise its taste.
\end{abstract}

\section{General instruction}

Sino-Japanese cultural exchanges have become increasingly frequent prompting a large number of Japanese cultural products into China via the internet; the most important is the Japanese animation products. Recently, the Chinese government also attaches great importance to this emerging cultural industries animation, because at this stage of China's animation industry is still in the emerging stage, and there is a big gap between Japan and other developed countries, the animation industry. Japan started in Asian culture industry is the earliest, especially the development of the animation industry with considerable strong local characteristics, the various aspects of Japanese film and television animation are touted by our young people, and gradually integrated into Chinese society. Based on Japanese television anime phenomenon spreading in our country, not only to investigate the Japanese film and television animation and its impact on the enjoyment of visual, auditory and other sensory aesthetic of the economy, the social dimension of our country, more in-depth from linguistics It analyzes the impact of its linguistic phenomena intercultural communication on Chinese.

Japanese anime buzzwords as a new source of social language, recently touted by the majority of youth groups, and gradually be brought into their daily lives. Japanese animation on the source language, our predecessors have done research, but mostly from the artistic angles anime carried out. Few based vocabulary, semantics, grammar and cultural linguistics and other related theories on the source animation on the network environment systematically study the language from the linguistic point of view, in order to strengthen the academic study of Chinese language network language animation, this article will use knowledge source for the popular
Japanese animation words were, multi-angle analysis of decency. Finally, the emergence of this phenomenon and put forward their own thinking.

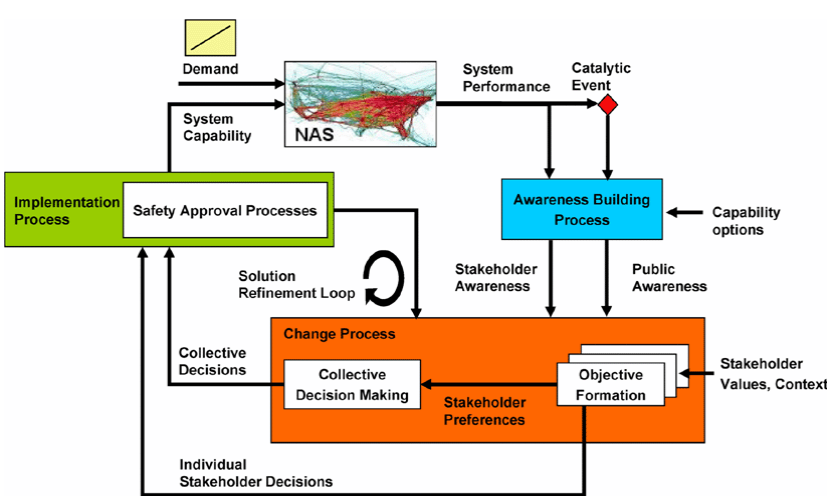

Figure 1. Implementation challenges for Japanese-original anime words systems

The text is written symbols of language recording system; it is the most important auxiliary communicative tool. It helps people to break through the language restrictions in time and space, so to expand the communicative function of language. With the development of human society, improve the technological level of long-distance communication and information exchange only in the form of letters already can not meet people's needs. The emergence is the Internet, to bring a new way of communicating, a new variant of the language - the language network. Largely solve the people exchanges require efficient, simple and so on, to meet the communication needs of people fast.

Internet language has broad and narrow sense; we put some new language phenomenon around computer technology and network exchange and the formation of, collectively known as the Internet language, which is a broad network language. It can be broken down into three 
categories: one is the network-related terminology, such as a mouse, hardware, software, browser, firewall; second category is network-related special terms, such as login, Internet cafes, users, hackers, broadband; the third category is the language and symbols of Internet users in chat or blog, forums, and paste it frequently use, such as top, sofa, bench, sell Meng, fans, $4 \mathrm{U}, \wedge \wedge \wedge$ and so on. The first class of network language and jargon on the same jargon are used in a professional field, while the second and third categories of network language is the language of life related to the Internet, it is a narrow network language. We need to study is the third type of network language, these words is that people in the network exchanges frequently used fresh, emerging a class of languages, and thus is called a network buzzwords.

\section{Network buzzwords features}

Network buzzwords used in the form of a keyboard input characters, numbers, letters, Roman letters, Japanese kana, punctuation, symbols, and other symbols of operation appears that Internet technology as the basis, the Internet as a medium to express some semantics, network communication for the purpose of language. Therefore, it has with the written, spoken different and unique place.

Network buzzwords also buzzwords in one, so its essential characteristic is "pop." "Pop" is "real time" and "dynamic". Therefore, there is a network buzzwords development process: to produce pop, pop to unpopular. In line with people's social communication requires a period of buzzwords to have a strong vitality, not in line with the will gradually be eliminated. It would appear promising buzzwords have two: one is to be absorbed, be absorbed into the general vocabulary Chinese. One is the disappearance of the course they will gradually be eliminated. This shows that the buzzword is "real-time", was eliminated just buzzwords history vocabulary. Network buzzwords as is "dynamic", it then disappeared from generation to popular or accepted, there is a long or short process; generally this popular procedure is relatively short-lived, because it has real-time, that is, in rapid Under the Internet, driven by some of the new, popular term on the Internet can be immediately spread of Internet users go. When it is used after a period of time, it will disappear. If it does not, its popular frequency reaches a certain level; people will lose their freshness, which eventually evolved into fairly generic common words. For example earlier "rookie" "Dinosaur," "white collar" "blue collar" "wage earners" and other network buzzwords, have now had their glory days, become a common word. Old things will always be replaced by new, older network buzzwords same will be replaced by new, as times change, the subjective and objective conditions for the survival of the old buzzwords will disappear, he lost the popular soil, and naturally they would cease to exist. At this era can reflect the new features of the new buzzwords too will produce, this is a dynamic process of development buzzwords transition from the old. For example, network language to describe a person lovely time, from "cute" to "kawaii," the popular earliest use to the current popular "Moe" is a very typical example.

Buzzwords is a highly creative language, is a modern human wisdom. Appears at the beginning, it always gives a new look, the feeling shines, this is one important reason for the popular buzzwords, especially network buzzwords. China's Internet user's mainly young people mainly, aged mainly between 21-35 years old. Features such groups of Internet users are quick-witted, curious, creative, attention to social popular things, flaunt personality, "has a very strong sense of anti-traditional, advocating innovation, from the traditional language syntax, semantics norms, standards constraints." Therefore, they dare to break the inertia of thinking style, form their own unique insights. Coupled may be published in the virtual space to conceal the identity of their own opinions and views, have a full right to speak, they are continuing to create a rich, vivid image words and expressions to express themselves, such as "God horses ( what) are the clouds, "" ginseng (life) cup (tragedy) "and other words such networks would highly infectious. In addition to the characteristics of Internet users among themselves, due to the progress of the times, people thought of liberation, the pursuit of individuality and efficiency of modern man seems unwilling to make the idea of the network so bland speak. They want to use the most simple words to express the time-saving richest, most vivid sense, but also highlights their personality and different, which reflects the contemporary creative network buzzwords.

The first need is communication: simple and quick, easy effort. With the rapid development of networks, the arrival of the information age, people increasingly prefer to use simple and clear language to express themselves, such as letters, numbers, etc. to convey information, common international letters, numbers can be expressed with a minimum of convey the wealth of information, so in this particular era was born of a number of lettered words, numbers, words. Online communication is actually in writing, spoken exchange rate. Oral communication course is the best, most concise communication tool, but the lack of specificity of the visual. Currently the network communication, we mainly keyboard input tool, according to certain input procedures. Keyboard input pinyin to input Chinese characters not only have this one form, also contains letters, numbers, the arithmetic symbols, each symbol in different languages have different sounds, such as "8" as a figure in the Chinese and English have different pronunciation, and even differences in Mandarin and dialects, such as people in some areas to read it as "fat" meaning "fortune."This gave us a simple symbol provides a rich voice material for quick and easy communication provided sufficient condition. In order to achieve writing speeds spoken, so some of the netizens will use shorthand symbols or acronyms, or create some new vocabulary. For example, "3KS (thanks abbreviations)" only need to type three times, but in the original word thanks input when you need to type 6; "OIC (oh, I see homonym 
representation I understand)," when in input only need to type three times, the original sentence is not punctuation, but also need to type 6; Again Pinyin input "Jiang Zi (" like this "homonym acronym)" To type 7, type the original phrase will have 9 times. Some networks such buzzwords not only impressive, but also reflect the characteristics of its simple and easy to write, greatly reducing the communication speed.

Language network has a large number of the biggest feature is the use of symbols to communicate. Or with a combination of symbols representative of expression, such as, $(* \wedge \ldots \wedge *),{ }^{\wedge}{ }_{-}^{\wedge}, \mathrm{O}\left(\cap_{-} \cap\right) \mathrm{O} \ldots \ldots$ and other means "happy smile"; (@_.@@) X_. X o $\left(>_{-}<\right)$o $\sim$..... such as performance, "sad, sad" facial expression; or using symbols words, such as "5201314" stands for "I love you for life" "+ U" means "Go" meaning: they represent an object or symbol combination, such as, $(\cdot \cdot)$ nnn represents a caterpillar, $-<_{-}<_{-}<@$ is a flat flower roses. We are very vivid glance. In addition, there is the ancient Chinese writing enabled or borrow foreign words, and so on the way. For example, nowadays very fire of network buzzwords "embarrassing" was originally an ancient Chinese word, and now being touted users, because it is very intuitive showed a man embarrassing, embarrassing the way, and as "Mei" presence in the ancient Chinese, "Kangxi": "stay with 喿 province, or a, pass for plum Herbal: stay, plum apricot class."But in today's buzzwords in network, it also has a new meaning, Internet users look directly shape pronunciation, read "foolishly, or double-stay" means "silly, stay, very naive" means. Like body with a source vocabulary to express abstract semantics, or other circumstances also need to use a lot of words to explain and describe, the network popular word sometimes can be more simple image but also no shortage of humor render out, and has a strong visual effect to the virtual network life adds some angry and realism.

\section{Dissemination in Japanese animation in China}

There is "anime Kingdom," said Japan, currently the world's largest exporter of animation production. Japanese animation film and television work in China spread of 30 years, with a gradual increase in the spread of technology, showing distinct stage characteristics: release from the official Internet TV to DVD and then released.

For the general audience in terms of Japanese anime is not yet a need for psychological, mass media (mass media mainly refers to the traditional forms of media, such as movies, television, newspapers and magazines, best-selling book, etc.) to provide animation film and television works must extent to meet their common needs. But for that loyal audience of Japanese anime film and television works in terms of the mass media to provide animation film has been completely unable to meet their needs, they strongly want to look for another way to solve this problem.
Officially entered China from the Internet to millions of households starting, the number of Internet users in China since 1997 after doubling every six months remained growth. Today, China's Internet users have reached a very large number, more than 400 million people. At the same time, with the development of mobile phones, mobile services, the Internet is more ubiquitous, deep into the lives of ordinary people into make people read online and online viewing more convenient, easy, and more without spending expensive cost far Japan to seek anime crossing. And it also includes all the way showing the animation film of this cultural product: text, pictures, audio and video. Thus, in the network's convenient, fast and cheap sexual attraction, the anime fans will choose the internet of course. Thus, downloading via a network or online to watch Japanese anime number of visitors also increased significantly.

\section{Network popular Japanese anime loanwords source classification}

With the popularity of the Internet, Japanese animation film to do a lot of influx in China. For the first time via the Internet to translate these incoming Japanese anime into Chinese available to the majority of Chinese animation fans, partially proficient in Chinese and Japanese anime fans spontaneously established online subtitle group, in the translation process, in order to pursue efficiency, they sometimes use the Japanese kanji homology characteristics of the Japanese kanji words copied from or slightly modified, thus creating a large number of Chinese and Japanese Loan Word. Only the pursuit of translation efficiency is not enough, in order to ensure the quality of translation, they will use transliteration, translation and other means to translate. Then these forms special, innovative Japanese loanwords can easily meet contemporary animation fans novelty, difference of mentality, as their common language. Anime in Japanese language borrowed words include two forms: Loan Word and transliterated words.

The relationship between language and society very closely, language can not be separated from social and independent existence. Stalin said: "To understand the language and its development law, we must take the history of language with social development, with the creation of the language, use the language of the people closely linked to the history of research." Language with human society is interdependent influenced. Whatever the language, will be affected by other different kinds of languages, even among its own internal dialects will influence each other, penetration. Thus, the famous American linguist Bloomfield once said: "Unlike traditional features called mainstream adoption, called the language of borrowing in the range borrow, borrow and borrow our cultural differences between the dialects, the former borrowed features come. to the same language area, which features borrowed from different languages. "From the lineage of view, belongs to the Sino-Tibetan Chinese, and Japanese belong to Altaic. Therefore, Chinese with Japanese vocabulary belongs cultural 
borrowing between borrowings. Now academics tend to the Chinese cultural definitions of words borrowed from other languages are: borrowing and varying degrees of "finished," the word from the alien language.

\section{Network popular Japanese anime loanwords source of inspiration to us}

Japanese animation culture through their own success enhance their influence in the international arena, we can also learn from them, we strive to improve the international influence of Chinese to improve China's comprehensive national strength. At any stage of a society, to improve the social cohesion of the country and the whole nation, language planning to do is to increase the frequency of the national language exchanges or the whole nation. In the previous stage of agricultural civilization, because each nation, each country link exchanges between each other is not close enough, so at that time just in terms of language planning issues in relation to a country. This will develop into modern humans, especially the era of economic globalization, any nation; any country can not rest on its laurels, and can not stay out of the tide of globalization. Therefore, an important task at this stage of language planning is to improve the country's national or native language influence.

In the context of today's globalization, cultural and ideological content has also become an important part of a national interest, and culture between the countries, comprehensive national strength has increasingly become an important parameter for the balance of interests and power. Cultural penetration and reverse osmosis are becoming an important part of the context of globalization of contention between the national interests. Currently English is rapidly expanding, linguistic hegemony prospects if you do not see this phenomenon later and blindly praise their guide language unified trend, welcomed it as the arrival of the world's common language, that this idea would be naive and harmful. States in order to enhance their own language in the international community's influence all have vigorously pursued a variety of language policy, mainly to improve the economic strength of the language. Economic power of language is a language refers to the percentage of gross national product in the region or country of the world's gross national product to use.

According to the data show that in 1977, the Englishlanguage economic power $34.6 \%$, Japanese economic power is only $8.2 \%$, in 2000, when English was $34.9 \%$, $11.4 \%$ for the rapid increase in Japanese. In these 23 years, the English language economic force grew only 0.3 percent; the Japanese grew by $3.4 \%$. This phenomenon can be seen, although the social status of English as an international language has not changed, but as a small Japanese language to enhance the international influence is enormous. This is thanks to the Japanese government's language promotion policy. Before World War II, the Japanese language promotion policy for war service, the purpose is to make the Japanese to become the lingua franca in Asia and become one of the tools of aggression. After World War II, the Japanese government aims to promote Japanese Japanese become an important means to make them with the world of cultural and economic cooperation. This policy relies on Japanese animation industry developed, strong economic strength and advanced technologies, has achieved remarkable results. In recent years, with the popularity of the Internet and youth groups love for Japanese animation, Japanese language economic strength will be further improved.

\section{References}

1. Peng Yubo. Some Features of Network Language. Rhetorical study, 4 (2001)

2. Lin Gang. Types and characteristics of the network terminology. Rhetorical study, 26 (2002)

3. $\mathrm{Li} \mathrm{Li}$. The nature and characteristics of the network words. Language Studies, 21 (2005)

4. Cao Guojun Comics - Animation - Cartoon • animation. Language knowledge, 7 (2006)

5. Chen Dandan, Chen Lin. Students analyze "otaku house female" phenomenon of Neijiang Normal University, 12 (2010)

6. Chu animation as a language in contemporary cinema, 3 (2010)

7. Gu Jiangping. Analysis of contemporary Japanese Loan Words on Chinese penetration. Kanji culture, 1 (2000)

8. Luo Yixian, party Wei Ying. On the influence of Japanese animation culture of our young people. Science garden, 6 (2008)

9. TURAL multimedia technology on Foreign Language Listening Course of Modern Languages (late), 05 (2006)

10. Feng. Foreign Language Teaching hear the application of multimedia teaching methods. Chinese education technology and equipment, 20, (2008) 